\title{
Improvements in Thermal Profiling Using High-Definition Fiber Optic Sensing
}

\author{
David Potter ${ }^{1}$, Andreas Stern ${ }^{2}$, Aida Rahim ${ }^{1}$ \\ ${ }^{1}$ Luna Innovations, Blacksburg, VA, U.S.A. \\ ${ }^{2}$ Luna Innovations, Pfullingen, Germany \\ potterd@lunainc.com, sterna@lunainc.com,rahima@lunainc.com
}

\begin{abstract}
Summary:
One of the main challenges of using distributed fiber optic sensing technologies is their sensitivity to both strain and temperature. This paper describes a new development in high-definition distributed fiber optic sensing (based on Rayleigh backscatter and OFDR instrumentation) that provides for the automatic compensation of mechanical strain when measuring distributed temperature, enabling broader adoption of the technology in embedded and surface mounted measurement applications.
\end{abstract}

Keywords: Temperature, fiber optic, strain, distributed

\section{Background and Motivation}

Fiber optic sensing (FOS) have become relatively common in a wide range of testing and monitoring applications, due to their many benefits and advantages, such as EMI immunity, passivity, reduced cabling and very small, flexible sensors. High-definition (HD) fiber optic sensors additionally deliver the highest spatial resolution for distributed measurements. For example, commercially available systems can resolve strain or temperature along a fiber with a gage pitch, or spacing, as low as $0.65 \mathrm{~mm}$.

One challenge of using most fiber optic sensing technologies comes from the fact that the sensors react to both mechanical strain and temperature. Therefore, when it is difficult to physically isolate strain from the fiber, accurate and reliable temperature measurements can be difficult in many applications. In particular, applications involving attaching or embedded longer sensor fibers to a surface with bends or curves can experience measurement errors due to strain.

This paper describes an important improvement to high-definition temperature sensing that compensates for strain and delivers more reliable and accurate temperature measurements when subjected to mechanical strain.

\section{Fiber Optic Sensing - Measurement Theory}

The temperature sensor is formed by standard off-the-shelf optical fiber composed of a monolithic fused silica core and cladding and a protective coating. An individual sensing fiber can be many meters in length and provide thou- sands of strain measurements at points distributed along its length. Measurements are made using the Rayleigh scatter in the fiber, a random but stable pattern of reflections inherent to each fiber as a result of small-scale nonhomogeneities in the fiber. This random pattern of reflections is unique to each fiber and constant for the life of the fiber, forming a reflection 'fingerprint'. Temperature changes experienced by the sensor results in variations of both its refractive index (due to the thermo-optic coefficient, dn/dT) and its length (due to the coefficient of thermal expansion, CTE). Both these effects result in an apparent stretching of this 'fingerprint', which translates to a shift in the spectral content of the 'fingerprint'.

Sensors are interrogated using optical frequency domain reflectometry (OFDR), an interferometric technique which can distinguish sensors or scattering points at different locations along the fiber. Figure 1 describes the basic OFDR network. Light from a swept-tunable laser is split between the measurement path and a reference path by a fiber optic coupler. Light

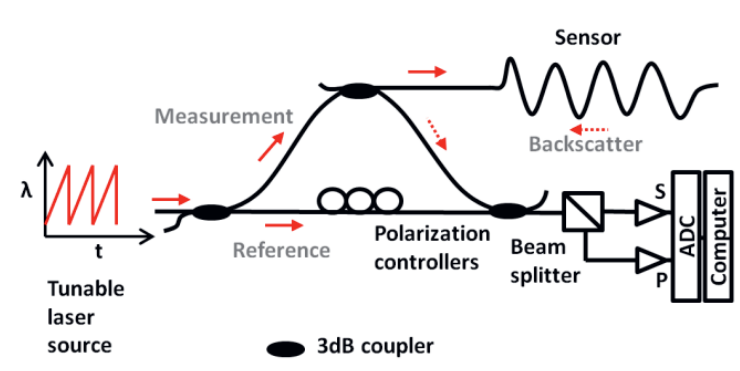

Figure 1. Basic OFDR system for measurement of temperature and strain in optical fiber 
reflected from the sensing fiber returns through the coupler and is recombined with light from the reference path. This combined signal then passes through a polarization beam splitter, which splits the light into orthogonal states recorded at the $S$ and $P$ detectors. A Fourier transform of these signals yields the phase and amplitude of the signal as a function of length along the fiber, i.e. the fiber 'fingerprint'.

To calculate temperature change, the spectral content of the fiber's 'fingerprint' is compared between the measurement and reference state. Complex Fourier transform data is windowed around a desired measurement location. An inverse Fourier transform of the windowed data gives the spectral content from a particular gage in the sensing fiber (which is crosscorrelated with the spectrum from the same location of fiber in a baseline state). Finally, the cross-correlated shift is converted to temperature change using a calibration coefficient. This process is repeated along the length of the fiber, forming a distributed measurement.

\section{Strain Compensation when Measuring Temperature with Distributed FOS}

This paper describes the effectiveness of an improvement to this OFDR system to essentially separate and remove the impact of strain on the measurement signal.

The high-definition strain-compensated (HDSC) system utilizes the advanced polarization capabilities of the described OFDR system, combined with specialty fiber-based temperature fiber, taking advantage of polarization effects, to decouple strain and temperature for more reliable and accurate temperature measurements.

\section{Verification of Strain Compensation}

In order to demonstrate the strain compensation capabilities of the HD-SC sensor, a $5 \mathrm{~m}$ fiber sensor was fixtured into a system, shown in Figure 2, which is able to selectively apply strain and temperature to different sections of the fiber optic sensor. The sensor is routed along a pulley system (configured to apply set amounts of strain) and then into a controllable temperature chamber. Therefore, there are sections of the fiber sensor that are subject to applied strain (at room temperature), elevated temperature and strain, and temperature only (loose fiber, no strain). A Luna Innovations ODiSI optical interrogator is used to acquire and compute the temperature measurements.

Figure 3 shows a summary of the results. In the regions where mechanical strain is applied to the sensor, the non-compensated (standard) temperature sensor registers very large errors which increase with the amount of applied

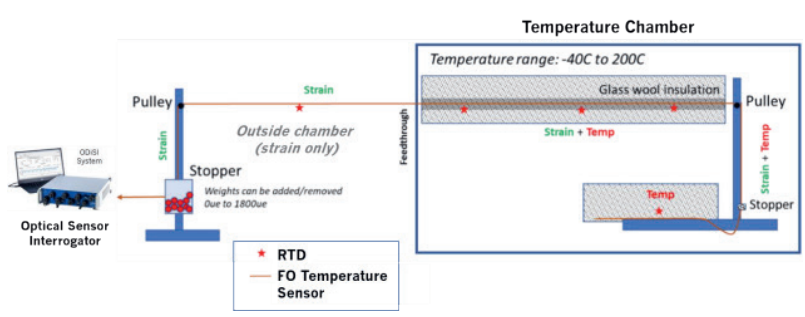

Figure 2. Experimental setup to verifiy strain compensation

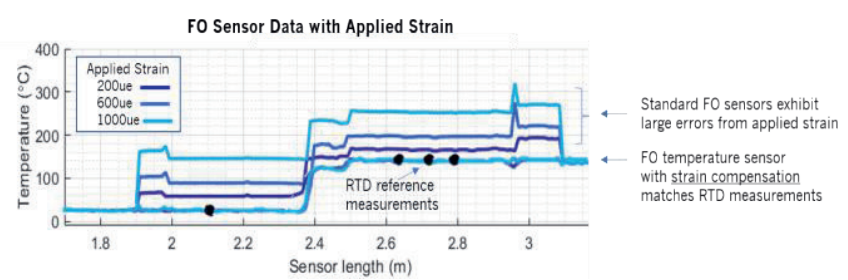

Figure 3. Experimental results, showing data with strain compensation and without

strain. However, the strain-compensated HDSC temperature sensor is largely unaffected and returns measurements that match the reference RTD measurement.

\section{Applications of High-Definition Temperature Measurements}

With increased accuracy and measurement reliability provided by the strain-compensation capability, HD temperature measurements should prove even more effective in key applications, such as:

- Monitoring of battery cell/packs and power electronics

- High-resolution thermal profiling of precision reactors

- In-situ monitoring of thermoplastic welding processes

The high-resolution thermal profiles, with hundreds or more distinct sensing points per meter of fiber, provides a unique measurement capability for these challenging applications.

\section{References}

[1] J. Bos, J. Klein, M. Froggatt, S. Sanborn, D. Gifford, Fiber Optic Strain, Temperature and Shape Sensing via OFDR for Ground, Air and Space Applications, Proc. SPIE 8876, Nanophotonics and Macrophotonics for Space Environments VII, September 24, 2013.

[2] A. Rahm, J. Pandher, N. Coppola, V. Penumetsa, M. van Tooren, In-Situ Monitoring and Control of Induction Welding in Thermoplastic Composites Using High Def Fiber Optic Sensors, CAMX Conference Proceedings, September 2019. 